\title{
La presencia del Quijote en los inicios de la enseñanza especial en Galicia
}

\author{
Ana RodríGuez DíAz* \\ Elisa Gavari StaRkiE*
}

No hay obra alguna en el mundo de que se hayan hecho tantas ediciones y traducciones como de El ingenioso hidalgo (...).

Hoy las ediciones se multiplican y abaratan, popularizándose, y poniéndolas al alcance de todos, para que no haya un español que no conozca el libro en que se ha retratado con tan admirable fidelidad nuestro espíritu nacional ${ }^{1}$

En 1905, el estado español organizó por primera vez los actos cervantinos para conmemorar el tercer centenario de la inmortal obra de Miguel de Cervantes, El ingenioso hidalgo Don Quijote de la Mancha. Universidades, institutos y escuelas participaron con diferentes eventos en la memoria de dicha efeméride. Los establecimientos de enseñanza especial, por entonces escasísimos en España $^{2}$, se sumaron a dicha celebración. Un ejemplo de ello es el festival que se celebró en la escuela gratuita de ciegos y niños pobres en la ciudad gallega de A Coruña, que preparó para este día un programa de actos muy completo, pese a las carencias y precariedad que acompañaba a este tipo de establecimientos. Cabe destacar que durante las primeras décadas del siglo XX solo el colegio de sordomudos y ciegos de Madrid constituía una escuela completa con profesorado para las enseñanzas generales, especiales y profesionales, con instalaciones

\section{* UNED.}

1. Liga de Amigos de la Coruña (1905: 4).

2. Molina Martín (1900: 455). 
adecuadas, material pedagógico especial y capacidad para un significativo número de alumnos ${ }^{3}$.

Lo cierto es que a principios del siglo XX todavía resultaba una novedad que los ciegos pudiesen leer. Así, en 1925, el director del Colegio Nacional de Sordomudos y Ciegos se preguntaba: «¿Será cierto, como afirma M. Perouze, profesor en l'Institution des Jeunes Aveugles de París ${ }^{4}$, que hay muchas gentes que no conciben que los ciegos tengan libros y puedan leerlos? $»^{5}$. Sin embargo, a pesar de su incapacidad visual, los ciegos eran capaces de leer, del mismo modo que la discapacidad del propio Miguel de Cervantes Saavedra, inválido del brazo izquierdo, no le había impedido escribir El ingenioso hidalgo don Quijote de la Mancha, obra inmortal que marcó el cenit de la novela ${ }^{6}$.

Desde 1825 existían libros para ciegos, y si bien el aprendizaje de la lectura y la escritura (así como de las otras enseñanzas) se consideraba un lujo para los invidentes ${ }^{7}$, las escuelas y colegios que velaban por la educación de estos alumnos consideraban la lectura un instrumento fundamental para la educación e integración de sus discípulos, fomentando la transcripción de libros para ciegos. Suponía una tarea laboriosa y costosa, que se complicaba aún más cuando se trataba de una obra tan extensa como esta novela cervantina. Pese a ello, en 1903, dos años antes de que los escolares ciegos pudiesen deleitar a los oyentes en el festival conmemorativo del tercer centenario del Quijote con la lectura de los pasajes cervantinos, un alumno destacado (y más tarde un excepcional profesor del colegio de sordomudos y ciegos de Santiago de Compostela) había comenzado, por propia iniciativa, la transcripción de la obra. No obstante, no era el único que emprendía esta monumental tarea en una fecha tan temprana: un profesor de la escuela de ciegos de Alicante, Francisco Just y Valentí («el primer ciego que trazó con la pauta y el pincel metálicos el gran libro de Cervantes $»^{8}$ ) llevaba más de una década dedicado a ello9. Y es que por su envergadura, la conversión de la inmortal novela de Cervantes al braille supuso todo un reto para los profesores de enseñanza especial cuando esta disciplina se encontraba en sus inicios.

3. Herraiz Gascueña(1995: 17).

4. L'Institution des Jeunes Aveugles de París fue la primera escuela para ciegos, fundada en París por Valentin Haüy en 1784.

5. González $(1925,18)$.

6. Aguado Díaz (1995: 103).

7. La educación de los ciegos era considerada como un lujo innecesario que no les serviría para encontrar una ocupación en el futuro y que además era una instrucción que requería de material y profesorado especial. Ballesteros y Fernández Villabrille (1862: 120).

8. Dorado Martín (1925: 1).

9. En 1887 Francisco Just ya había transcrito una copia de la obra. "Noticias locales y regionales", El Alicantino: Diario Católico, 23 de enero de 1894, núm. 1782, 3. 


\section{LA LECTURA DE CERVANTES POR LOS CIEGOS: FESTIVAL CONMEMORATIVO DEL TERCER ANIVERSARIO DEL QUIJOTE EN LA ESCUELA GRATUITA DE CIEGOS Y NIÑOS POBRES DE A CORUÑA}

«... Ahora digo, dijo á esta sazón D. Quijote, que el que lee mucho y anda mucho, ve mucho y sabe mucho» ${ }^{10}$.

La conmemoración del centenario de la obra de Miguel de Cervantes El ingenioso hidalgo Don Quijote de la Mancha se convirtió en uno de los mayores eventos sociales de la primera mitad del siglo XX. Las instituciones de todas las provincias españolas se sumaron a los actos de la celebración:

Se celebrarán grandes fiestas nacionales para solemnizar el 3. ${ }^{\text {er }}$ centenario de la publicación del Quijote: «Serán erigidos varios monumentos en las ciudades donde ha habitado en las que figuran en su obra inmortal, se colocará su busto en todas las escuelas de España; se publicará una obra conmemorativa de su obra maestra; se organizarán festejos en los que figuren todos personajes de D. Quijote, exposiciones especiales y representaciones teatrales $»^{11}$.

Las provincias gallegas conmemoraron el centenario con la misma solemnidad que lo hizo el resto de España. "Mal podía dejar la Atenea de Galicia de contribuir con su homenaje a la noble empresa de enaltecer la memoria del héroe de Lepanto, del inmortal hidalgo D. Miguel de Cervantes Saavedra (...)"12. Concretamente en la provincia de A Coruña tuvieron lugar fiestas literarias en el Instituto General y Técnico y en la Escuela Normal de Maestras, una velada en la Escuela gratuita de ciegos y niños pobres, una exposición en la Escuela de Artes e Industrias, una fiesta académica en la Escuela de Comercio y un concurso literario en el Colegio Budén ${ }^{13}$.

La Junta Oficial, constituida a principios de 1904 con el fin de organizar el tercer centenario de la publicación del Quijote, había acordado que las fiestas y actos solemnes previstos en tal ocasión tuviesen lugar el 7, 8 y 9 de mayo de 1905. Estos días se declararon festivos en los centros oficiales de enseñanza. Se disponía además, lo siguiente: "En las universidades, institutos, escuelas especiales, escuelas normales y en las de niños y niñas podrá organizarse ese acto con absoluta independencia y adaptándolo a las condiciones y elementos de cada establecimiento"14.

10. Cervantes Saavedra (1825: III, 223).

11. «El Quijote», La Correspondencia Gallega: diario de Pontevedra, marzo de 1904, núm. 423, 2.

12. Cosfrán de Llarvi (1905: 3).

13. Chaparro Domínguez (2011: 103).

14. Anuario legislativo de Instrucción Pública correspondiente a 1905, Madrid, Sección de Estadística de Instrucción pública de la Subsecretaría del Ministerio, 1906, p. 55. Lo cierto es que a principios del siglo XX, sobre todo a raíz del año 1905 con las conmemoraciones del tercer centenario, la novela del Quijote, se introduce en el ámbito escolar, en servicio de un proyecto nacional a la búsqueda de un símbolo de identidad que respaldase el país. En este sentido, consultar Guereña (2008: 145-190). 
Siguiendo las directrices de dicha disposición, la escuela gratuita de ciegos y niños pobres situada en el Campo de la Leña, . $^{\circ} 8$, en la ciudad de A Coruña, organizó un festival para celebrar el tercer centenario del Quijote. Esta escuela inició su andadura en 1893, cuando el presbítero José María Salgado instauró en A Coruña una escuela dedicada a la enseñanza de los niños pobres. En dicha fundación habían cooperado dos jóvenes sacerdotes y un grupo de adolescentes que contaban con la autorización de sus padres, dada su corta edad, para encargarse de la enseñanza de los escolares. Dos años después, en 1895, se destinó en este establecimiento un departamento a la instrucción de los ciegos ${ }^{15}$. A principios del siglo XX, dicha escuela representaba uno de los escasos establecimientos que se dedicaban en España a la educación de estos alumnos, y figuraba como la segunda institución dedicada a la enseñanza especial en Galicia. La primera fue el colegio regional de sordomudos y ciegos inaugurado en la ciudad de Santiago de Compostela el 1 de junio de 1864.

El director de la escuela, José María Salgado, aspiraba a proporcionar a sus alumnos una instrucción que estuviese a la altura del el Colegio de Sordomudos y Ciegos de Madrid, modelo de los establecimientos de enseñanza especial durante la mayor parte de las décadas del siglo XX. La penuria y escasez que por entonces era la tónica general de los colegios y escuelas que se fueron inaugurando a finales del XIX y principios del XX no impidió que el programa de enseñanza se fuese ampliando y perfeccionando ${ }^{16}$. Los alumnos demostraban sus habilidades con notable éxito, acudiendo a diferentes certámenes y festivales. En 1905, la escuela se encontraba en condiciones de rendir su especial homenaje a la obra cervantina. A continuación se reproduce en su totalidad la invitación al festival organizado en el establecimiento, en la que se describen las distintas actuaciones programadas para el evento ${ }^{17}$ :

\section{ESCUELA GRATUITA DE CIEGOS Y NIÑOS POBRES EL CAMPO DE LA LEÑA, LA CORUÑA $n .{ }^{\circ} 8$ \\ Sr. D.}

El director de este centro instructivo tiene el honor de invitar a V. para

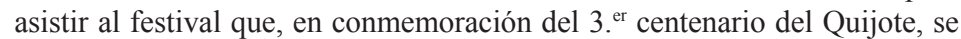
celebrará en los salones del citado establecimiento los días, 7, 9 y 11 del corriente de cinco a siete de la tarde.

Con este motivo saluda a V. reiterándole los sentimientos de su consideración más distinguida, Att. S. S.

José M. ${ }^{a}$ Salgado

15. Rodríguez Díaz (2014: 260).

16. Véase el programa de enseñanza completo de la escuela correspondiente al año 1904 en Archivo Histórico Universitario de Santiago de Compostela, Fondo Universitario: Colexios Privados, Caixa, SH 228, expediente 19, Escuela gratuita de ciegos y niños pobres, 1904.

17. Archivo Histórico Municipal, A Coruña. Invitación-programa da "Escuela gratuita de ciegos y niños pobres" para asistir ao festival conmemorativo do $3^{\circ}$ Aniversario do "Quijote" (imp.). Referencia K. caja 977, exp. 10. 


\section{1. ${ }^{a}$ PARTE:}

1. Himno a Cervantes. Letra del profesor D. José García. Música de D. Luciano Caño, profesor de ciegos, coreado por los alumnos.

2. ${ }^{\circ}$ Breve narración o noticia sobre la vida y obra de Cervantes, con especialidad del Quijote. Diálogo en verso entre dos pastores sobre el mismo tema, por los niños Enrique Villar y Gustavo Rey.

\section{2. ${ }^{\mathrm{a}}$ PARTE:}

1. Sinfonía, por el cuarteto de la Escuela de ciegos.

2. Poesía a Cervantes, declamada por el niño de seis años de edad Recaredo Mesa García, e imprecación a Sancho Panza, poesía burlesca declamada por el alumno Juan Rivero. Ovillejos, entre el alumno Andrés Sánchez y los de la sección superior, y el soneto recitado por el alumno Enrique Ulloa.

3. ${ }^{\circ}$ «La vuelta de Escarramán....» ${ }^{18}$

Juguete cómico lírico en prosa y verso de personajes representado por los alumnos ciegos Claudina Fernández y Ramón Puga y los niños José Ponte, Julio Castro, Gumersindo González. Coro formado por los alumnos de una y otra clase. Letra original de D. José García Taboada y música de Luciano Caño y Lamas, ambos profesores del establecimiento. Decoraciones construidas expresamente por el joven pintor escenógrafo, D. Camilo Díaz. La parte lírica será acompañada al piano por el alumno José Villar Longueira. Amenizarán los intermedios con escogidas composiciones al piano algunas distinguidas señoritas de la localidad.

La Coruña, mayo de 1905

En la revista gallega, días después de la celebración del festival, podía leerse lo que sigue:

Las escuelas populares gratuitas y de ciegos y niños pobres también contribuyeron a celebrar el tercer centenario del Quijote. En esta última, o sea, la escuela de ciegos y niños pobres que tan filantrópicamente sostiene y dirige el presbítero José María Salgado, infatigable propagador del bien y de la instrucción, dióse una fiesta por lo demás simpática ${ }^{19}$.

Entre los participantes en el festival se encontraban los alumnos ciegos de la escuela, que leyeron poesías, declamaron y cantaron algunos pasillos cómicos, ejecutándose en los intermedios números musicales. Las decoraciones, pintadas para el teatro, representaban un salón estilo Luis XV, otro románico y una calle del siglo XVI. Los decorados fueron muy aplaudidos, al igual que el Sr. Salgado, organizador de este festival, y los profesores D. José García y D. Luciano Caño.

18. Escarramán, figura como uno de los protagonistas más pintorescos de la cultura y literatura del siglo de oro, que responde al arquetipo de un valeroso rufián, un desvergonzado, un bandido, idealizado o un auténtico pícaro «que aparece repetidamente en las obras desde finales del siglo XVI a principio del XVIII», Begué, Delage y Sola (2006: 326).

19. «Las fiestas cervantinas», Revista gallega: semanario de literatura e intereses regionales, 13 de mayo de 1905, núm. 530, 4. 
Figura 1. Plano de la escuela donde se organizó el festival, Archivo histórico universitario de Santiago de Compostela, Fondo Universitario: colexios privados, Caixa, SH 228, expediente 19, Escuela gratuita de ciegos y niños pobres, 1904.

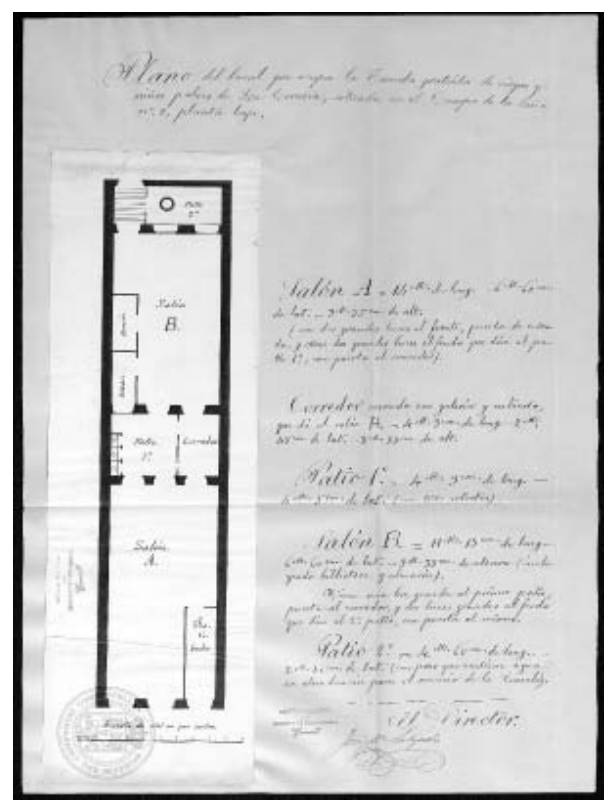

\section{EL QUIJOTE ESCRITO PARA LOS CIEGOS: EL PRIMER QUIJOTE EN BRAILLE. GALICIA POSTERGADA}

Las propuestas para solemnizar el tercer centenario de la publicación del Quijote fueron variadas: actos oficiales, exposiciones, cabalgatas, festivales, obras de teatro, etc. Entre estas propuestas se encontraba la de fundar una Biblioteca Cervantina en Alcalá de Henares. En dicha biblioteca, donde se reunían una importante colección de manuscritos dedicados al insigne novelista, figuraba un ejemplar con una parte del Quijote escrita en braille.

Desde que fué iniciada la idea de solemnizar el tercer centenario de la publicación del «Quijote», la junta local allí nombrada tomó con gran calor y mucho empeño los antiguos propósitos de formar en la ciudad del Henares un Museo Biblioteca Cervántico (...) .Hay también en el Museo Biblioteca algunas curiosidades como una reproducción única de parte de un ejemplar también único del «Quijote para ciegos» ${ }^{20}$.

El enorme mérito de haber escrito en braille una parte del ejemplar de Quijote (único, hasta el momento, en su género), utilizando un punzón y la pauta de escritura que usaban los ciegos, se debió a D. Francisco Yust y Valentí21,

20. El centenario del Quijote: El museo biblioteca Cervántico de Alcalá de Henares», El Imparcial: Diario Liberal, 19 de abril de 1905, núm. 13 672, 2.

21. El autor del Quijote para los ciegos», $A B C, 3$ de noviembre de 1905, 8. 
profesor de la escuela de ciegos de Alicante desde finales del siglo XIX. El insigne maestro llevaba veinticuatro años dedicado a esta ardua tarea, que lamentablemente no pudo concluir.

En mayo de 1925, en los principales titulares de la prensa madrileña y en la sección telegráfica de un buen número de periódicos de provincia, aparecía la noticia de que el Colegio Nacional de Sordomudos y Ciegos «había tenido la humanitaria idea en favor de la cultura y de la alegría de los ciegos» de hacer una edición del Quijote en caracteres adecuados a la lectura de los invidentes. Con motivo de la festejar el inicio de la impresión, se celebró una festividad en el que no faltó el busto de Miguel de Cervantes. A dicha solemnidad acudieron varias personalidades destacadas del mundo de la política y la cultura. Se pronunciaron discursos en los que se elogiaba la novela cervantina y a su genial autor; se resaltó, además, la importancia de la obra que se emprendía y el orgullo del Colegio Nacional de Sordomudos y Ciegos en realizarla.

El periódico La Vanguardia hizo mención al acto de entrega de la primera edición del Quijote para la lectura de los ciegos. La ceremonia estuvo presidida por el subsecretario de Instrucción Pública, y asistieron -entre otras ilustres personalidades- el alcalde, los miembros del patronato, la duquesa de Parcent, los señores Aguado y Giménez Encina. El acto lo inauguró el director del colegio, que explicó la labor de la institución, añadiendo que uno de sus alumnos, el ciego Domingo Rodríguez, lo había escrito valiéndose de una máquina especial suiza.

Cabe señalar que desde la adopción del braille como método de escritura para los ciegos fueron varios los procedimientos que se ensayaron para la impresión de libros en este sistema. En sus inicios estos manuales eran manuscritos, y la primera impresión que se realizó se hizo de la manera usual en los libros para videntes, por lo que resultaba excesivamente caro. Más tarde se utilizó para ello un sistema consistente en escribir con punzón de acero y martillo sobre plancha de cinc los caracteres braille. El procedimiento requería demasiado esfuerzo y era muy lento. Posteriormente se comenzaron a utilizar máquinas, pero tenían el inconveniente de que su precio era muy elevado, por lo que las bibliotecas para ciegos estaban constituidas mayoritariamente por libros manuscritos. Por otra parte, la diferencia entre un volumen normal y uno en braille obligaba a economizar el papel ${ }^{22}$ :

Un volumen de 300 páginas de impresión corriente, que tiene 10 centímetros de largo, 11 de ancho y poco más de uno y medio de grueso y pesa 370 gramos, puesto en Braille, es un libro en ocho ó diez volúmenes de un total de 1.000 páginas de 29 por 22 centímetros, que pesa cinco kilos; es decir, un volumen y un peso, respectivamente, 600 y á veces mayor.

La máquina ideada y utilizada por el Colegio Nacional de Sordomudos y Ciegos en la impresión del primer Quijote en braille, según apunta el que por 
entonces era su director, reunía muchas ventajas con respecto a los procedimientos usados hasta el momento en la impresión de los libros para ciegos. El director del Colegio Nacional describía así este método:

Consiste en obtener, á máquina, mediante una Piteh, corrientemente empleada para mecanografiar en Braille, un poco modificada, clichés estereotípicos con latón fino, que se logran con la rapidez misma de la mecanografía usual y resisten tiradas mucho mayores que los de papel. De este modo conseguimos hacer libros muy baratos y hemos podido emprender la magna empresa de hacer la primera edición en braille del Quijote ${ }^{23}$.

En el acto de presentación de la primera edición del Quijote en braille, que tuvo lugar en Madrid, se había efectuado una demostración de este tipo de escritura con dicha máquina. El ciego Domingo Rodríguez escribió varios párrafos del Quijote, dictados alternativamente por la duquesa de Parcent, por el señor García de Leaniz y por el alcalde: "Los caracteres quedaron impresos en una hoja metálica y fueron leídos por la niña Emilia Larrea, que es la más joven del establecimiento"24. De las páginas leídas, se habían realizado copias sobre papel por procedimiento mecánico.

Los periódicos que recogieron en sus titulares la ceremonia aplaudieron esta iniciativa, calificándola de interés nacional, y añadieron que esa era la primera edición en España. Sin embargo, si bien la edición de este ejemplar del Quijote en braille sería la de mayor alcance oficial (tras haber sido verificada, entre otras personalidades, por el ministro de Instrucción Pública y la edición con mayores expectativas de difusión ${ }^{25}$ ), la iniciativa de transcribir a braille el Quijote había sido emprendida años atrás.

Un diario alicantino se apresuró a desmentir la noticia que aparecía en los periódicos madrileños, testimoniando que la primera edición del Quijote para la lectura de ciegos no era la que se exhibió en la fiesta de referencia, recordando a los periodistas madrileños que hacía más de veinte años Franciso Just y Valentí, un modesto profesor auxiliar de la escuela de ciegos y director más tarde de esta, daba cima a una edición del Quijote para la lectura de los ciegos $^{26}$.

El diario gallego El Compostelano también desmintió la noticia de que la primera edición del Quijote correspondiera a la editada en 1925, y reclamó el lugar que le correspondía al colegio de sordomudos y ciegos de la ciudad gallega de Santiago de Compostela, al haber realizado con anterioridad que el

23. González (1925: 19).

24. «En el colegio de ciegos» en la sección de información nacional, La Vanguardia, 24 de abril de 1925,14 y 15.

25. En la ceremonia celebrada en Madrid en el año 1925, se llegó a decir: «Así podrán leer ella hermosa obra de Cervantes los niños ciegos de la América Latina», en Montero Pérez, Francisco, «Un lamentable olvido: Para la intelectualidad alicantina», Diario de Alicante, 28 de abril de 1925, núm. 4047, 1.

26. Montero Pérez, Francisco, «Un lamentable olvido: Para la intelectualidad alicantina», Diario de Alicante, 28 de abril de 1925, núm. 4047, 1. 
colegio madrileño una versión del Quijote para ciegos. El autor de este escrito, Ortiz Novo, acusó a la capital española de desentenderse de todo lo provinciano, pasando inadvertidas las iniciativas que desde las demás provincias españolas se emprendían:

No es afán de apagar entusiasmo sin restar esplendor al acto que acaba de celebrarse en el colegio de sordomudos y ciegos de la Corte, lo que hoy mueve mi pluma. Tampoco es fobia hacia lo que ostenta el marchamo madrileño... Es únicamente legítimo el orgullo de gallego y de santiagués y noble deseo de recabando a lo que Galicia le pertenece emplazar la verdad en el lugar que le corresponde ${ }^{27}$.

En 1916 Ortiz Novo, el mismo autor de las informaciones aparecidas en El Compostelano, que por aquel entonces se encontraba estudiando en la universidad compostelana, había publicado en el periódico El Eco de Santiago del 28 de abril una crónica con el título "El Quijote para los ciegos, precioso y raro ejemplar". En dicho artículo se mencionaba a los autores de la obra: el director del colegio de sordomudos y ciegos de Santiago de Compostela, José Cruz Letamendi, y un profesor, del mismo establecimiento, Enrique Rodeiro. Esta ardua labor se había iniciado el 4 de agosto de 1915:

En efecto con paciencia benedictina, durante 185 sesiones, cada una de hora media y dos horas de duración, fueron los señores, Letamendi, y Rodeiro, levantando el monumento que figurará en la exposición del Centenario de la muerte de Cervantes, consta aquel de 229 páginas a folio". En cada sesión escribieron de seis a dieciocho páginas en el sistema Braille. Cada página contiene el texto de trece líneas del original. La obra se reparte en más de veinte voluminosos tomos; En cada uno de ellos, aparece una ilustración en relieve relativa al contenido de los capítulos transcritos. Exteriormente, la encuadernación de cada uno de los tomos, era lujosa ${ }^{28}$.

El Colegio de Sordomudos y Ciegos de Santiago de Compostela, primero de los instituidos en España de acuerdo a los mandatos de la Ley de Instrucción Pública de 1857, era un colegio cuya organización era digna de destacar, si se compara con los establecimientos contemporáneos. Contaba con una gran biblioteca en braille literaria y musical, con obras de diferentes materias, algunas de ellas escritas sin el uso de medios mecánicos por Enrique Rodeiro, destacado alumno y más tarde ilustre profesor del colegio ${ }^{29}$. Gracias a la práctica y habilidad de dicho maestro se concluyó esta gran obra: "El punzón manejado por sus hábiles manos iba dejando en los blancos y dobles pliegos a través de

27. Ortiz Novo, «El Quijote para los ciegos. Galicia postergada», El Compostelano: Diario Independiente, 11 de mayo de 1925, núm. 1548, 1.

28. Ortiz Novo, «El Quijote para los ciegos, precioso y raro ejemplar», El Eco de Santiago, 28 de abril de 1916, núm. 7186, 1.

29. En el año 1903, Enrique Rodeiro, había comenzado a copiar el Quijote. Montoro Martínez (1995: 615). 
los surcos de la rejilla los abultados puntos que ingeniosamente combinados, forman las letras y los signos en relieve de esta escritura ingeniosa que hoy se prestigia al facilitar a los ciegos el famoso libro"30.

En una conferencia pronunciada en Santiago por un distinguido literato y catedrático que versaba sobre la inmortalidad de Cervantes en el cielo del arte también se constató la existencia de una versión en braille del Quijote en el colegio gallego, anterior a la editada en Madrid:

... Y además estando próximas las fiestas de su centenario a las que esta vetusta ciudad donde en cada callejuela se encuentra un trozo histórico, contribuyó en gran escala con dos obras de universal transcendencia, primero el estudio crítico del teatro de Cervantes por el catedrático de Derecho, doctor Armando Cotarelo que empezó ya a surtir efectos tanto en el mundo literario, como en beneficio de su persona y, "El Quijote para los ciegos" por el director de dicha escuela, D. José Cruz Letamendi, con lo cual desde ahora podrán estos infelices deleitarse con la obra que hizo inmortal a su autor y elevó a la nación española a la cumbre del arte literario ${ }^{31}$.

Según el testimonio de la prensa ${ }^{32}$, en 1916 en el colegio regional de sordomudos y ciegos de Santiago de Compostela se contaba ya con un ejemplar del Quijote para ciegos y, como intuía acertadamente el autor de la crónica aparecida en El compostelano ${ }^{33}$ y testimoniaba el diario alicantino, no era el único existente. No obstante, que hubiese o no algún manual en braille más de la obra no era importante. Lo realmente significativo es que en las primeras décadas del siglo XX, tanto los niños como los adultos ciegos, al igual que los videntes, podían disfrutar de la lectura de las aventuras del ingenioso hidalgo, transcrita íntegramente al braille; un tributo muy especial en memoria del denominado príncipe de los ingenios, que el colegio gallego de sordomudos y ciegos de Santiago de Compostela había rendido en una fecha muy temprana:

Miguel de Cervantes ¡Salve, genio inmortal!

Autor famoso de la más grande y peregrina historia;

que alcanzaste del mundo la victoria

luchando con la pluma valeroso.

Bien quisiste en tu honor joh gran coloso!

¡astro radiante de la hispana gloria!

30. Ortiz Novo, «El Quijote para los ciegos, precioso y raro ejemplar», El Eco de Santiago, 28 de abril de 1916, núm. 7186, 1.

31. Goleat, Isidrín, «La conferencia en los Luises», Gaceta de Galicia: Diario de Santiago. Decano de la prensa de Compostela, 14 de febrero de 1916, núm. 13, 2.

32. Cabe destacar que el año 1916 también, se edita en Madrid un catálogo por la imprenta municipal de la Biblioteca cervantina, en caracteres Braille en la primera Biblioteca pública de ciegos de la escuela de ciegos y sordomudos. El director de la escuela y promotor de la idea fue Eduardo Molina. En este catálogo que comprendía 471 ejemplares, se encontraba un ejemplar de cada una de las obras de Cervantes siendo el resto de miscelánea cervantina. Serrano Vélez (2005: 87).

33. Ortiz Novo, «El Quijote para los ciegos. Galicia postergada», El Compostelano: Diario Independiente, 11 de mayo de 1925, núm. 1548, p. 1. 
Un tributo rendir a tu memoria y a tu hidalgo manchego el ingenioso. No pudiendo admirar, de luz privado, tu libro incomparable y sin segundo, en letras de relieve y adornado lo habré yo de escribir; y si en el mundo place a Dios que mi aliento no se agote, los ciegos leer podrán tu don Quijote ${ }^{34}$.

\section{BIBLIOGRAFÍA CITADA}

Aguado Díaz, Antonio (1995). Historia de las deficiencias. Madrid: ONCE.

Ballesteros, Juan Manuel y Fernández Villabrille, Francisco (1862). Curso elemental de instrucción de ciegos y de sordomudos. Madrid: Imprenta del Colegio Nacional de Sordomudos.

Begué Alain, Delage, Agnès y Sola, Christel (ed.) (2006). Investigaciones recientes sobre la literatura del Siglo de Oro. Homenaje a Julián Durán. Seminario de la Casa de Velázquez (Madrid, 3-4 de mayo de 2004) (Criticon 97-98). Toulouse: Casa de Velázquez, Instituto Cervantes y Presses Univertaires du Mirail.

Chaparro Domínguez, María Ángeles (2009). «La prensa española ante la publicación de la primera parte del Quijote (2005)», Anales Cervantinos. XLI, pp. 285-315.

Chaparro Domínguez, María de los Ángeles (2011). La construcción social de la cultura: el Quijote como icono cultural a través de las representaciones mediáticas de las celebraciones del III y IV centenario. Tesis Doctoral. Madrid: Universidad Complutense de Madrid. Accesible en: http://eprints.ucm.es/13784/1/T33158.pdf

Cervantes Saavedra, Miguel de (1825). El ingenioso hidalgo Don Quijote de la Mancha. París: Librería de Cormon y Blanc. Tomo III.

González, Anselmo (1925). «Libros para ciegos como los hacen y como los leen», La Esfera, 600, (4 de julio), pp. 18-19.

Guereña, Jean-Louis (2008). «¿Un icono nacional? La instrumentalización del Quijote en el espacio escolar en el primer tercio del siglo XX», Bulletin Hispanique. 110/1, pp. 145-190.

Herraiz Gascueña, Mariano (1995). Aproximación a la educación especial española del primer tercio del siglo $X X$. Murcia: Compobell.

Liga de Amigos de la Coruña (1905). El centenario del Quijote en Galicia. La Coruña: Imprenta de Pedro Ferrer.

López Navalón, Manuel (1867). Memoria sobre el estado actual del Colegio de SordoMudos y de Ciegos de Santiago. Mejoras de que es susceptible para su futuro progreso $y$ prosperidad. Santiago de Compostela: Imprenta Manuel Miras.

Miñana, Juan José (2005). «Poemas de Francisco Just y Valentí», Revista Esperanza. 3 Accesible en $<$ http://revistaesperanza.com/ $>$ [consulta el 9/10/2014].

Molina Martín, Pedro (1900). «Los Institutos de sordomudos y ciegos», La Escuela Moderna, Revista Pedagógica Hispano-Americana, 111, pp. 438-454.

Montoro Martínez, Jesús (1995). Los ciegos en la historia. Madrid: ONCE.

34. Poesía en homenaje a Miguel de Cervantes de Francisco Just y Valentí, profesor de la escuela de ciegos de Alicante a finales del siglo XIX. Miñana (2005). Accesible en: $<$ http://revistaesperanza. com/> [consultado el 9/10/2014]. 
Rodríguez Díaz, Ana (2014). «La escuela de ciegos del Campo de la Leña, A Coruña: los inicios de la enseñanza especial en Galicia», Cuadernos de Estudios Gallegos. 61/127, pp. 241-278.

Serrano Vélez, Manuel (2005). Locos por el Quijote. Zaragoza: Caja de Ahorros y Monte de Piedad de Zaragoza, Aragón y Rioja.

Recibido: 17 de febrero de 2015

Aceptado: 02 de septiembre de 2015

\title{
Resumen
}

Este artículo pretende mostrar la presencia de la obra de Cervantes, Don Quijote de la Mancha, en los únicos establecimientos existentes en Galicia a principios del siglo XX, dedicados a la enseñanza especial. Conforme a dicha pretensión, se hará referencia a dos efemérides significativas en la difusión de la obra. La primera corresponde a la conmemoración del tercer centenario del nacimiento del Quijote, celebrado en el año 1905 en la escuela gratuita de ciegos y niños pobres de A Coruña. En este establecimiento se organizaría un festival muy completo según consta en el contenido del programa, que se ha recuperado del archivo histórico municipal de dicha ciudad. El segundo evento se refiere a la presentación de la novela de Cervantes escrita en braille. Todo un reto para los profesores de los establecimientos de enseñanza especial de la época, que el colegio de sordomudos y ciegos de Santiago de Compostela, primer colegio para la enseñanza de estos alumnos que se establece en España a efectos de la ley de instrucción pública de 1857 , asumirá tempranamente.

Palabras clave: Miguel de Cervantes, El Quijote, enseñanza especial, braille, Galicia.

Title: The Presence of Don Quijote in the Beginnings of the Special Education in Galicia.

\begin{abstract}
This article aims to show the presence of the work of Cervantes, "Don Quijote de la Mancha", in the unique establishments existing in Galicia at the beginning of the 20th century, dedicated to special education. According to this claim, reference is made to two significant anniversaries in the dissemination of the work. The first one corresponds to the commemoration of the third centenary of the birth of the Cuisse. In this Establishment one would organize in the year 1905, in the free School of blind persons and poor children of To Corunna. A very complete festival as appears in the content of the program, which has recovered of the historical municipal file of the above mentioned city. The second event, refers to the presentation of the novel by Cervantes, written in Braille. Quite a challenge for teachers in special schools of the time, as the College of deaf and blind from Santiago de Compostela, the first school for the teaching of these students, which is established in Spain for the purposes of the law of public instruction of 1857, will assume early.
\end{abstract}

Keys words: Miguel de Cervantes, "Don Quixote", Special education, braille, Galicia. 